\title{
La planète Saturne \\ Mythologie, astronomie, éthique cosmique
}

\author{
Jean-Pierre Levet \\ Université de Limoges
}

De nos jours, la lointaine planète Saturne fascine ceux qui l'observent avec des instruments optiques appropriés par la somptueuse beauté de ses anneaux. Ces derniers étaient inconnus des Anciens, qui pourtant percevaient dans cet astre errant, pour eux le plus éloigné de la terre ${ }^{1}$, des enseignements importants.

On se propose de les analyser et de les replacer dans la perspective d'une pédagogie éthique liée à la contemplation des cieux nocturnes.

Les étoiles et les planètes offraient aux Anciens des repères chronologiques naturels, censés annoncer les grands cycles de la vie annuelle. On peut ainsi être certain que leur observation n'était pas réservée aux seuls astronomes.

Ainsi, dans les Travaux et les Jours, Hésiode invite-t-il les agriculteurs à établir le calendrier de leurs tâches en prenant les Pléiades comme repères majeurs : " commencez à moissonner au lever des Pléiades, filles d'Atlas, et semez à leur coucher $»^{2}$. Il apporte une précision, dont toute personne est capable de vérifier l'exactitude par une simple observation : "pendant quarante nuits et quarante jours, elles demeurent invisibles, mais l'année s'avançant, elles redeviennent visibles au moment où on aiguise le fer $»^{3}$.

On remarquera que ces étoiles portent un nom emprunté à la mythologie et connu de tous, puisque l'aède, en l'utilisant, se sent dispensé d'apporter des précisions concernant leur nombre, leur disposition et leur position dans l'étendue du ciel. Ce nom renvoie à un mythe, dont on est en droit d'estimer que nul ne l'ignore. L'indication de la filiation, qui renvoie à Atlas, confirme bien que l'on passe de l'astronomie à la mythologie sans qu'il soit besoin d'avancer des explications.

Les noms des principaux corps célestes ${ }^{4}$ et des constellations dans la composition desquels ils entrent font donc partie de la culture commune dans ce qu'elle a à la fois de plus savant (elle est mentionnée par l'aède), de plus populaire (elle est familière aux laboureurs). En elle se trouvent également les grands récits de la mythologie, sinon Hésiode n'aurait eu aucun intérêt à mentionner Atlas à propos d'un groupe d'étoiles.

La voûte céleste et les grandes histoires dont elle évoque le souvenir font donc pour ainsi dire partie des référents traditionnels reconnus par les Grecs dans leur vie de tous les jours.

\footnotetext{
${ }^{1}$ Uranus, Neptune, Pluton ne sont pas observables à l'œil nu ; seuls de puissants instruments, lunettes et télescopes, ont permis, à l'époque moderne ( $\mathrm{au} \mathrm{XX}^{\circ}$ siècle pour ce qui est de Pluton), de découvrir ces planètes.

${ }^{2} 383-384$.

3 385-387.

${ }^{4}$ On pourra consulter, en ce qui les concerne, Richard H. Allen, Star Names. Their Lore and Meaning, New York, 1963, ou encore André Le Boeuffle, Les noms latins d'astres et de constellations, Paris, $1977=\mathrm{Le}$ Boeuffle (1977).
} 
Or deux excellents ouvrages récents de vulgarisation ${ }^{5}$ ont rappelé à juste titre que se manifestait un enseignement moral fondamental derrière ce que nous considérons comme des légendes, mais qui correspondait à des enseignements quasi historiques pour les Anciens, empruntés à des zones de contact entre ce qui était présenté comme vérité et comme une fiction porteuse de vérité6.

Or la base de l'éthique grecque repose sur la distinction entre la démesure (hybris) et la justice (dikê). Elle est clairement exposée au vers 213 des Travaux d'Hésiode : "Persès ${ }^{7}$, écoute la justice et ne laisse pas se développer la démesure ».

Ou bien, en effet, l'homme se plie à la loi morale, dont les dieux, et en particulier Zeus, sont les auteurs et les garants, ou bien il la transgresse et commet alors un acte de démesure puisqu'il s'affranchit, lui simple mortel, par l'effet d'un libre choix relevant d'un orgueil insensé, de la volonté des maîtres de l'Olympe.

Toute faute relève de l'hybris dans son principe. Comme telle, elle implique un aveuglement et une sanction qui ne manquera pas de frapper tôt ou tard le coupable.

Certes, il existe des degrés dans l'hybris et ce sont les responsables des plus grands crimes, comme Prométhée, ou des actes les plus présomptueux, comme Phaéton, que la mythologie rend tristement célèbres par les catastrophes qu'ils ont engendrées, pendants des bienfaits apportés aux hommes par un héros authentique tel qu'Héraklès.

Les actions scélérates ou bienfaitrices, les châtiments et les récompenses, les réparations d'injustices subies constituent le fondement des métamorphoses astrales, qui fournissent donc la matière de ce que l'on pourrait appeler un grand livre d'éthique cosmique, dont l'accès est ouvert à tous.

Les moralistes trouvent ainsi un champ d'éducation morale facile à exploiter.

Mais les savants se tournent aussi vers le ciel pour en faire le héraut de la recherche d'une vie droite, c'est-à-dire en harmonie avec l'univers, expression du divin, l'hybris étant une rupture de cette harmonie, que l'homme est invité à placer au centre de sa vie.

Ainsi Ptolémée, dans le prologue de son Almageste, estime-t-il que la contemplation de ce qui est à la fois le vrai et le beau dans l'univers représente la meilleure formation éthique possible, si l'homme comprend qu'il doit vivre moralement et socialement en accord avec le rythme parfait du mouvement des corps célestes.

Science théorique par nature, la mathématique, centrée sur la recherche des harmonies dans les domaines qui sont les siens, arithmétique, géométrie, astronomie et musique, a vocation à inspirer les sciences pratiques que sont la politique, l'économie et surtout l'éthique : «la mathématique, plus que n'importe quelle autre discipline, rendra les gens perspicaces pour examiner l'honnêteté parfaite dans les actions et dans les conceptions morales. En montrant la similitude qui existe entre le bien et ce qui est perçu des objets divins $^{8}$, en montrant la bonne ordonnance du cosmos, l'harmonie, la simplicité naturelle, elle suscite chez ceux qui s'intéressent de près à cette beauté divine une passion pour elle, elle les habitue à se conformer à elle, elle les accoutume à faire entrer dans leur âme une semblable disposition, comme si elle les inspirait $»^{9}$.

Contempler l'harmonie universelle doit susciter l'envie de l'imiter, d'en faire la loi de l'existence, de vivre en harmonie avec le monde et avec les autres, avec un idéal où le vrai, le beau et le bien ne sont qu'une seule et même réalité.

\footnotetext{
${ }^{5}$ Marie-Françoise Serre et Pierre Bourge, Histoires d'étoiles. Les merveilleuses légendes du ciel de l'Antiquité, La Mesnière, 1991 = Serre (1991) ; Luc Ferry, La sagesse des mythes, Paris, 2008.

${ }^{6}$ Voir sur ce point, par exemple, Théogonie 27 et 28.

${ }^{7}$ À travers son frère, Hésiode, au cœur de son poème didactique, s'adresse ici à tout être humain.

${ }^{8}$ Les astres.

${ }^{9}$ Almageste I, 1.
} 
Pour le savant qui se fait à sa manière philosophe, le ciel devient donc, comme pour le mythologue, un outil primordial de formation éthique, que le cosmos met à la disposition de tous, car si comprendre suppose l'acquisition de la science, admirer, et donc percevoir tout ce qui est harmonie, se révèle à la portée de tous, sans qu'il soit nécessaire de recourir aux calculs approfondis et aux démonstrations géométriques complexes.

Comme les noms des astres et les récits populaires dont ils sont évocateurs, les mouvements apparents parlent à l'âme humaine et l'éduquent en suscitant en elle une aspiration au bien, qui la mettra en paix avec elle-même, avec les autres et avec l'univers.

Les planètes, elles aussi, comme les étoiles, apportent aux hommes des messages dans les mêmes conditions, c'est-à-dire par leurs déplacements et par ce que symbolisent les noms qui leur ont été attribués.

Mercure (Hermès), Aphrodite (Vénus), Mars (Arès), Jupiter (Zeus) sont des divinités du panthéon classique, aux fonctions et aux caractères connus de tous. Nul n'ignore, par exemple, que Mars est le dieu de la guerre et du sang versé, mais aussi de la jeunesse, qui est appelée à fournir la grande masse des combattants, et du printemps, saison où débutent, après l'interruption de l'hiver, les nouvelles campagnes. Chacun de ces noms constitue donc, pour quiconque tourne ses regards vers les astres concernés, une évocation des principaux dieux et de tout ce que l'on peut savoir d'eux. Jupiter, par exemple, rappelle la toute-puissance du maître de l'Olympe et sa volonté de châtier les auteurs d'actes de démesure.

Saturne, en grec l'astre de Kronos (Kronou astêr), renvoie à une génération divine antérieure à celle sur laquelle règne Zeus ${ }^{10}$.

Si les gens du peuple ignorent les effets que les astrologues lui attribuent sur la santé ou le destin en raison de ses qualités, que seule leur science leur permet de définir et d'interpréter, comme le fait Ptolémée dans son Tetrabiblos ${ }^{11}$, ils n'ignorent ni la lenteur de son cycle (globalement trente années, précisément environ vingt-neuf ans et demi) ni ce que signifie son nom.

À ce dernier est lié le souvenir de l'âge d'or, qui est situé sous le règne du père de Zeus (epi Kronou) ${ }^{12}$. En ces temps heureux, les hommes vivaient comme des dieux, ignorant les peines et la misère, insensibles au vieillissement, épargnés par les maladies et les maux, non soumis à la dure loi du travail, puisque la terre leur fournissait d'elle-même, dans sa fécondité, tout ce dont ils avaient besoin, pacifiques, engagés dans des fêtes perpétuelles et des banquets sans fin, ils connaissaient bien la mort, mais celle-ci n'avait rien d'effrayant, car elle était plus une sorte de sommeil, dont ils se réveillaient métamorphosés en génies bienfaisants, qu'une fin de la vie telle que la subiront les mortels des générations suivantes, appelés à descendre dans $1^{\prime}$ Hadès $^{13}$.

Le mythe hésiodique des races ne nous apprend pas pourquoi l'âge d'or eut une fin. En revanche, le récit parallèle de Pandore ${ }^{14}$ nous informe de ce qui s'est passé. C'est à la suite d'un double crime de démesure commis par Prométhée au détriment de Zeus que ce dernier fit fondre sur l'humanité les innombrables malheurs qui l'accablent sans cesse, le jour comme la nuit, sans qu'il lui soit possible de leur échapper.

De sa naissance à sa mort, l'homme, qui doit durement travailler pour produire ce dont il a besoin pour se nourrir, en supporte le poids terrible, voué à la souffrance et aux peines qui le consument jusqu'à son dernier jour.

\footnotetext{
${ }^{10}$ Voir, par exemple, Hésiode, Théogonie, 453 sq.

11 Voir, par exemple, Tetrabiblos, I, 4.

${ }^{12}$ Les Travaux et les Jours, 111.

${ }^{13}$ Les Travaux et les Jours, 109-126.

${ }^{14}$ Les Travaux et les Jours, 42-105.
} 
La leçon qui est ainsi donnée trouve une place dans toutes les circonstances de l'existence. Elle explique ce qu'est le mal, à savoir une punition collective infligée à la suite d'actes de démesure, et enseigne que la condition humaine n'a pas toujours été ce qu'elle est.

Elle constitue donc un rappel permanent de ce qu'est l'exigence de droiture morale, de vertu, de respect du divin, et de ce que sont les conséquences funestes du vice, si bien qu'elle est à méditer à chaque instant et plus particulièrement toutes les fois qu'un choix s'impose à la conscience entre le bien et le mal. La voie de ce dernier paraît avantageuse, alors qu'elle se révèle à terme nuisible, voire catastrophique. Pour ardu qu'il soit, l'autre chemin, celui de la justice, est toujours profitable. Il faut donc bien réfléchir, avant de déchaîner, par ignorance ou par sottise, ou pis encore par vice, le châtiment qui suit inéluctablement la démesure ${ }^{15}$.

Voilà donc ce sur quoi est appelé à méditer quiconque, dans le grand poème, si l'on peut recourir à ce mot, de l'éthique céleste, tourne, il est vrai bien après l'époque où vécut Hésiode, son regard vers l'astre de Kronos, ainsi appelé seulement depuis Aristote ${ }^{16}$ et nommé auparavant Hêlios, c'est-à-dire « soleil », comme cela est rappelé dans l'Épinomis ${ }^{17}$ de Platon.

Mais la perception, acquise évidemment à l'œil nu, fait apparaître immanquablement la faible luminosité de cette planète, bien moins intense que celle du brillant Jupiter, si bien que le nom primitif de la planète pose un sérieux problème ${ }^{18}$.

André Le Boeuffle, suivi par M.-F. Serre et P. Bourge, fait état d'une source babylonienne. La planète, dans l'astronomie orientale, portait le nom du dieu Nin-Urta, «qui avait reçu, en tant que divinité planétaire, le nom de SAG-USCH (sémitique Kaimanu), « le stationnaire $», ~ «$ astre de justice et d'équité $» \ldots{ }^{19}$.

Par la lenteur apparente de son mouvement régulier, la planète se transformait en symbole de stabilité, d'équilibre, de modération, et par la suite de justice.

Mais pourquoi donc l'avait-on d'abord appelée Astre du Soleil, ce qu'aucune réalité astronomique ne justifie?

Elle était conçue comme le soleil de la nuit, sans que son faible éclat puisse en quoi que ce soit expliquer cette appellation. C'est donc dans les connotations morales qui lui sont attachées qu'il faut tenter de trouver la solution du problème posé.

Le soleil, pendant la journée, représente la lumière qui observe le monde. Pour les mortels, il est comme l'œil de Zeus, auquel rien ne peut échapper ${ }^{20}$. Mais les êtres vivants et le cosmos peuvent-ils être laissés sans surveillance lorsque son éclat disparaît ? Un autre observateur doit prendre son relais. Ce veilleur ne peut être que la plus éloignée des planètes, parce qu'elle peut projeter sa lumière sur les autres. Elle «domine $»^{21}$ pour ainsi dire le monde.

Cette fonction rejoint l'image de justice qui lui est également associée. La justice est lente, comme le mouvement de l'astre, mais elle finit toujours par triompher.

D'autre part, sa position peut laisser supposer qu'elle joue le rôle également physique, et pas seulement moral, de régulateur du ciel nocturne. Elle serait alors le fondement de l'ensemble de l'harmonie céleste, son organisateur et superviseur. Les Grecs, d'autre part, ont joué sur le nom de Kronos, qu'ils ont rapproché du terme (Khronos) qui désigne le temps.

Ainsi cette planète, si l'on examine son nom ancien (soleil de la nuit) et celui qui lui a été donné par la suite (astre de Kronos), avant d'être rapproché du Temps, symbolise-t-elle à

\footnotetext{
${ }^{15}$ Les Travaux et les Jours, 213-218.

${ }^{16}$ Métaphysique, 1073 b.

17987 c 5.

${ }^{18}$ On consultera la notice, p. 126, de l'édition de l'Épinomis dans la Collection des Universités de France (par A. Diès et E. des Places, Paris, 1956, avec bibliographie); on se reportera aussi à Serre (1991), p.142-143, et à Le Boeuffle (1977), p. 245-246, avec également une bibliographie.

${ }^{19}$ Le Boeuffle (1977), p. 246.

${ }^{20}$ Les Travaux et les Jours, 105.

${ }^{21}$ Serre (1991), p. 141.
} 
elle seule à la fois ce que les astronomes admirent dans le cosmos et qu'ils souhaitent transposer dans le domaine de l'éthique (l'ordre, l'harmonie, au demeurant parfaitement perceptibles même par les non initiés, simples spectateurs de la beauté de l'univers), et ce que les moralistes attachent à l'idée de Justice et à celle de Temps, fondues l'une et l'autre dans Kronos.

Ainsi le spectacle cosmique, inséparable de la culture mythique que l'attribution de noms aux constellations et aux planètes astres a indissolublement associée à sa contemplation, est-il porteur d'un message éthique accessible à tous et de nature à assurer à chacun une formation morale complète, qu'une seule règle résume : il faut se détourner de la démesure et pratiquer la vertu, qui est harmonie de l'homme avec lui-même, avec ses semblables, avec la société dans laquelle il vit, avec le monde divin et avec le cosmos entier, où se perçoivent conjointement le vrai et le beau, image unique, en leur union, du bien.

Les astres, complétés par les légendes et les mythes liés à leurs noms, sont porteurs d'un message dans lequel l'homme grec, avec sa culture héritée d'une enfance bercée par ces récits, découvre le sens du réel et celui de l'existence.

Dans cette multitude de corps célestes, Saturne dispose d'un statut particulier, puisque, à elle seule, elle porte un condensé de tout ce que la contemplation du ciel peut apprendre à l'homme.

Elle est bien, pour l'esprit en quête de vérité, un soleil qui l'éclaire du plus profond de la nuit, d'une clarté faible et pâle certes, mais véritablement lumineuse pour qui, dans le monde de la civilisation grecque, savait en saisir toute la signification, comme s'il la découvrait en lisant dans le plus grandiose «livre » de philosophie qu'il était alors possible d'imaginer et de consulter en toute simplicité.

Saturne, l'astre de Kronos, est peut-être le plus bel objet céleste et didactique de l'astroéthique, au point de rencontre de l'astronomie mathématique de l'harmonie et de l'onomastique astromythologique des Anciens, de la Mésopotamie au bassin méditerranéen..

RÉSUMÉ

Dans le Prologue de l'Almageste, Ptolémée, en tant qu'astronome et mathématicien, donne des leçons de philosophie ou, pour ainsi dire, d'astro-philosophie morale. Elles sont fondées sur le concept d'harmonie.

Les noms grecs des étoiles et des planètes renvoient à de nombreux héros légendaires, dont les bonnes ou les mauvaises actions sont racontées avec leurs conséquences dans de nombreux mythes populaires, connus de tous, si bien que tous les êtres humains, jeunes ou vieux, riches ou pauvres, peuvent en permanence contempler dans le ciel nocturne quelque chose qui ressemble aux pages illustrées d'un grand livre cosmique de morale qui serait facilement lisible.

Les connotations diverses liées à la planète Saturne (Kronos en grec) telles qu'elles sont étudiées dans cette contribution montrent d'importants et intéressants exemples d'astrophilosophie, d'astro-mythologie et d'astro-éthique. 
In the Prologue of the Almagest, Ptolemy, as an astronomer and as a mathematician, gives lessons of philosophy, or, so to speak, of moral astrophilosophy. They are based on the concept of harmony.

The greek names of stars and planets refer to many legendary heroes, whose good or bad actions are told with their consequences in many popular myths known by everybody.

Thus, all the human beings, whether they are young or old, wealthy or poor, can every night behold in the nocturnal sky something looking like the illustrated pages of a big and cosmic book written for their moral education and easily legible.

The various connotations attached to the planet Saturn (called Kronos in greek) and studied in this paper show important and interesting examples of astrophilosophy, astromythology and astroethics. 